\title{
Chronic inflammation of the prostate type IV with respect to risk of prostate cancer
}

\author{
Antonio B. Porcaro ${ }^{1}$, Emanuele Rubilotta ${ }^{1}$, Aldo Petrozziello ${ }^{2}$, Claudio Ghimenton ${ }^{3}$, \\ Filippo Migliorini ${ }^{1}$, Stefano Zecchini Antoniolli ${ }^{1}$, Vincenzo Lacola ${ }^{1}$, Carmelo Monaco ${ }^{1}$, \\ Pierpaolo Curti ${ }^{1}$, Stefano Cavalleri ${ }^{1}$, Romeo Pianon ${ }^{1}$, Walter Artibani ${ }^{1}$ \\ ${ }^{1}$ Urologic Clinic, ${ }^{2}$ Geriatric Medicine/Endocrinology, ${ }^{3}$ Pathology, University Hospitals, Ospedale Policlinico \\ and Ospedale Civile Maggiore, Azienda Ospedaliera Universitaria Integrata, Verona, Italy.
}

\section{Summary Background: Chronic inflammatory infiltrate (CII) might be involved in prostate cancer (PCA) and benign} hyperplasia (BPH); however, its significance is controversial. Chronic inflammatory prostatitis type IV is the most common non cancer diagnosis in men undergoing biopsy because of suspected PCA.

Objective: To evaluate potential associations of coexistent CII and PCA in biopsy specimens after prostate assessment. Design, setting, and participants: Between January 2007 and December 2008, 415 consecutive patients who underwent prostate biopsy were retrospectively evaluat$e d$. The investigated variables included Age (years) and PSA (ug/l); moreover, CII+, glandular atrophy (GA+), glandular hyperplasia $(\mathrm{GH}+)$, prostate Intraepithelial neoplasm (PIN+), atypical small acinar cell proliferation $(A S A P+)$ and $P C A$ positive cores $\left(P_{+}\right)$were evaluated as categorical and continuous (proportion of positive cores). Outcome measurements and statistical analysis:

Associations of CII+ and PCA risk were assessed by statistical methods.

Results and limitations: In the patient population, a biopsy core positive for PCA was detected in $34.2 \%$ of cases and the rate of high grade PCA (HGPCA: $b G S \geq 8$ ) resulted $4.82 \%$. CII+ significantly and inversely associated with a positive biopsy core $P+(P<0.0001 ; O R=0.26)$ and HGPCA $(P=0.0005 ; O R=0.05)$. Moreover, the associations indicated that patients with coexistent CII+ on needle biopsy were $74 \%$ less likely to have coexistent PCA than men without CII+ as well as 95\% less likely to have HGPCA in the biopsy core than men without coexistent CII+. There were limits in our study which was single centre and included only one dedicated pathologist. Conclusions: There was an inverse association of chronic inflammation of the prostate type IV and risk of PCA; moreover, HGPCA was less likely to be detected in cancers associated with coexistent CII. In prostate microenvironment, prostate chronic inflammation may be protective; however, its role in PCA carcinogenesis remains controversial and needs further research.

KEY WORDS: Prostate; Prostate cancer; Prostate-specific antigen; Prostate biopsy; Chronic inflammation; Biopsy Gleason score.

Submitted 23 December 2014; Accepted 31 March 2014

\section{INTRODUCTION}

Chronic inflammation plays an important role in human carcinogenesis $(1,2)$. Development and progression of cancer might be related to reactive oxygen and nitrogen species developing in tissue microenvironment after related damage and regeneration. Prostate cancer (PCA) carcinogenesis has also been related to chronic inflammation. Presence of chronic inflammatory infiltrate (CII) has been detected in PCA specimen from prostatectomies, transurethral resection of the prostate (TURP) and transrectal ultrasound (TRUS) biopsies $(3,4)$.

Literature investigations suggest that CII might be involved in chronic diseases of the prostate including PCA and benign prostatic hyperplasia (BPH) (5).

The prostatitis syndromes have been classified in four categories by the National Institutes of Health (NIH) (6). The last category, named type IV, has been coded as asymptomatic inflammatory prostatitis which is diagnosed in patients who have no history of genitourinary tract pain complaints, but undergo prostate biopsy for evaluation of possible PCA because of elevated serum prostate-specific antigen (PSA) level. As a result, chronic prostatitis is the most common non cancer diagnosis, based on histological criteria, in these men. Since the significance of CII in prostate specimens with and without cancer is still unclear and controversial, we evaluated the association, if any, of coexistent CII and PCA in patients undergoing TRUS biopsies after prostate assessment.

\section{MATERIAL AND METHOdS}

Between January 2007 and December 2008, we retrospectively evaluated 475 men referred to our institute for prostatic biopsy because of increased serum PSA and/or abnormal digital rectal exam (DRE). PSA was measured by immuno-radiometric test $(2-4 \mathrm{ug} / \mathrm{ml})$ and abnormal DRE findings were as follows: diffusely hard prostate, discrete firm area, irregular contours or prominent lobe asymmetry. Patients with DRE findings associated with painful prostate were excluded. The 14-core TRUS guided prostate biopsy technique was routinely performed and additional cores were taken with a lesion on either TRUS or DRE was evident. For each biopsy core, the dedicated pathologist systematically assessed the following issues:

No conflict of interest declared. 
(i) PCA and its grade according to the Gleason Score system (biopsy Gleason score: bGS); (ii) prostatic Intraepithelial neoplasia (PIN); (iii) chronic Inflammatory Infiltrate (CII); (iv) glandular atrophy (GA); (v) atypical small acinar cell proliferation (ASAP); (vi) glandular hyperplasia (GH). Atypical adenomatous hyperplasia (AAH), since not systematically assessed, was not included in the present analysis. Chronic inflammation criteria included the following findings: (i) inflammatory cell infiltrate within the stroma of the prostate; (ii) inflammatory cell infiltrate composed predominantly of lymphocytes with admixed plasma cells; (iii) peri-glandular distribution of the inflammatory cell infiltrate. Criteria excluding a diagnosis of chronic inflammation of the prostate type IV were as follows: (i) sheets of neutrophils around and within the glands; (ii) granulomatous prostatitis. A diagnosis of chronic inflammation of the prostate type IV (6) was carried out after excluding other types of inflammation.

\section{Statistical analyses}

The variables were evaluated as both categorical and continuous; moreover, the histological ones were coded as proportion of the number of positive cores and were labelled as CII+, GA+, GH+, PIN+, ASAP+ and P+, respectively. Summary statistics of population, subpopulations (with or without PCA) and relative groups was computed. Student t-tests were used to compare subpopulations and relative groups. In populations and subpopulation of patients, $\mathrm{X} 2$ tests were used to evaluate associations of CII+ with Age at the first quartile (Q1), $\mathrm{PSA}$ at $\mathrm{Q} 1, \mathrm{GA}+, \mathrm{GH}+, \mathrm{ASAP}+, \mathrm{PIN}+, \mathrm{P}+, \mathrm{bGS} \geq 8$ and DRE. Moreover, to evaluate the strength of associations, the odds ratio (OR) and relative $95 \%$ confidence interval (95\%CI) were also computed. CII+ independent associations with Age, PSA, GA+, GH+, ASAP+, PIN+, P+ and $\operatorname{DRE}(1=$ abnormal, $0=$ normal $)$ were evaluated by multivariate regression analysis in the population and subpopulations of patients. Because of the high level of correlation between $\mathrm{CII}+$ and $\mathrm{GA}+$ (correlation coefficient $=$ $0.49, \mathrm{P}<0.0001$ ), the multivariate independent associations of bGS were separately evaluated for CII+ and GA+. All tests were two-sided, with a significance level of 0.05.

\section{RESULTS}

After removing cases with incomplete data or excluding criteria, 415 of the 475 cases were able to be evaluated. Overall clinical characteristics of population and subpopulations with relative groups are reported in Table 1. Compared with cases without PCA, the PCA subpopulation was significantly older at diagnosis (69.23 years vs. 66,56; P < 0.0007); less likely to have larger CII+ $(0.06$ vs. $0.38, \mathrm{P}<0.0001), \mathrm{GA}+(0.16$ vs. $0.50, \mathrm{P}<0.0001)$ and $\mathrm{GH}+(0.01$ vs. $0.10, \mathrm{P}=0.0002)$; but more likely to have higher PSA serum levels $(29,6 \mathrm{ng} / \mathrm{ml}$ vs. $13,69, \mathrm{P}=$ $0.02)$ and higher $\mathrm{PIN}+(0.07$ vs. $0.03, \mathrm{P}=0.001)$. In the subpopulation without PCA, the group without CII+ was less likely to have larger $\mathrm{GA}+(0.44$ vs. $0.58, \mathrm{P}=0.001)$, but more likely to have larger proportion of $\mathrm{GH}+(0.14$ vs. $0.07 ; \mathrm{P}=0.06)$. However, in the PCA subpopulation, the CII+ group was significantly older ( 72.6 years vs.
68.10; $\mathrm{P}=0.003)$, more likely to have larger proportion of $\mathrm{GA}+(0.33$ vs. $0.11 ; \mathrm{P}=0.0003)$, but less likely to have greater PSA values (11.2 vs. $36.10 \mathrm{ug} / \mathrm{l} ; \mathrm{P}=0.04$ ) as well larger proportions of $\mathrm{P}+(0.25$ vs. $0.42 ; \mathrm{P}<0.0001)$. The group with $\mathrm{bGS} \leq 6$, showed lower proportions of $\mathrm{P}+$ than $\mathrm{bGS}=7(0.25$ vs. $0.48 ; \mathrm{P}<0.0001)$ and $\mathrm{bGS} \geq 8$ $(0.25$ vs. $0.65 ; \mathrm{P}<0.0001)$ as well as of ASAP+ than bGS $\geq 8$ (0.01 vs. $0.00 ; \mathrm{P}=0.01)$; interestingly, the $\mathrm{bGS} \leq 6$ group was more likely to have increased proportions of CII+ than $b G S=7(0.17$ vs. $0.07 ; \mathrm{P}=0.02)$ and $b G S \geq 8$ (0.17 vs. $0.005 ; \mathrm{P}<0.0001)$; moreover, significantly increased GA+ proportions were detected in the $\mathrm{bGS} \leq 6$ group than $b G S \geq 8$ (0.22 vs. $0.03 ; P<0.0001)$. The group with $\mathrm{bGS} \geq 8$ was more likely to be detected with increased proportions of $\mathrm{P}+$ than the $\mathrm{bGS}=7$ group $(0.65$ vs. $0.48 ; \mathrm{P}=0.04)$, but less likely to have increased proportions of $\mathrm{CII}+(0.005$ vs. $0.07 ; \mathrm{P}=0.008)$ and $\mathrm{GA}+$ (0.03 vs. $0.14 ; \mathrm{P}=0.01)$ than the $\mathrm{bGS}=7$ group.

Table 2 and Figure 1 show the associations of CII+ with the investigated variables in population and subpopulations of patients. CII+ was detected at a rate of $45.06 \%$ in the population, $26.65 \%$ in the subpopulation with PCA and $44.32 \%$ in the subset without PCA. Age $\leq$ Q1 resulted 61.67 years in population, 64.37 in the PC subpopulation and 60.93 in the subset without PCA. CII+ inversely associated with Age in the PCA subpopulation ( $\mathrm{P}<$ 0.0001; OR = 0.40). Total PSA serum levels $\leq \mathrm{Q} 1$ were $4.99 \mathrm{ng} / \mathrm{ml}$ in the population, $4.71 \mathrm{in}$ the PCA subpopulation and 5.14 the other subset. CII+ inversely associated with $\mathrm{PSA} \leq \mathrm{Q} 1$ in the population $(\mathrm{P}=0.002 ; \mathrm{OR}=0.47)$ and subpopulation without PCA $(\mathrm{P}=0.005 ; \mathrm{OR}=0.44)$. $\mathrm{GA}+$ was detected at a rate of $58.3 \%$ in the population, $38.7 \%$ in the PCA subpopulation and 68.5 in the other subset. CII+ directly associated with GA+ in the population $(\mathrm{P}<0.0001 ; \mathrm{OR}=5.14)$, $\mathrm{PCA}$ subpopulation $(\mathrm{P}<$ $0.0001 ; \mathrm{OR}=5.34)$ and subset without $\mathrm{PCA}(\mathrm{P}<0.0001$; $\mathrm{OR}=3.77$ ). A biopsy core positive for PCA was detected in $34.2 \%$ of the population. CII+ inversely associated with a positive biopsy core $\mathrm{P}+(\mathrm{P}<0.0001 ; \mathrm{OR}=0.26)$. In the PCA subpopulation, CII+ inversely $(\mathrm{P}=0.0004 ; \mathrm{OR}=$ 0.28 ) associated with a proportion of positive cores larger than the median $(\mathrm{P}+>0.33)$. The rate of high grade PCA (HGPCA: bGS $\geq 8$ ) resulted $4.82 \%$ in the population and $14.08 \%$ in the PCA subpopulation. CII+ inversely associated with HGPCA in both population $(\mathrm{P}=0.0005$; $\mathrm{OR}=$ 0.05 ) and subpopulation $(\mathrm{P}=0.05 ; \mathrm{OR}=0.13)$. An abnormal DRE was detected in $30.84 \%$ of the patient population and inversely associated with $\mathrm{CII}+(\mathrm{P}=0,01)$; however, the association was weak $(\mathrm{OR}=0.58)$ and was not confirmed in the two subpopulations.

The independent and multivariate associations of CII+ and bGS are reported in Table 3. In the population of patients, CII+ associated with GA+ $(\mathrm{P}<0.0001), \mathrm{GH}+(\mathrm{P}$ $=0.02)$ and $\mathrm{bGS}(\mathrm{P}=0.05)$; moreover, the association was positively related to GA+ (regression coefficient, $b=$ $0.38)$, but negatively related to $G H(b=-0.16)$ and $b G S$ $(b=-0.01)$. In the analysis excluding $G A+, b G S$ positively associated with $\mathrm{P}+(\mathrm{P}<0.0001 ; \mathrm{b}=9.85) ; \mathrm{PIN}+(\mathrm{P}$ $=0.0004 ; \mathrm{b}=2.67)$ and Age $(\mathrm{P}=0.02 ; \mathrm{b}=0.02)$, but inversely with $\mathrm{CII}+(\mathrm{P}=0.003 ; \mathrm{b}=-0.68)$. In the analysis excluding $\mathrm{CII}+$, bGS directly associated with $\mathrm{P}+$ $(\mathrm{P}<0.0001 ; \mathrm{b}=9.77)$, PIN+ $(\mathrm{P}=0.007 ; \mathrm{b}=2.58)$ and 
Age $(P=0.02 ; b=0.02)$, but inversely with AG+ $(\mathrm{P}<0.002 ; \mathrm{b}=-0.68)$. In the PCA subpopulation, $\mathrm{CII}+$ directly associated with $\mathrm{GA}+(\mathrm{P}=0.001 ; \mathrm{b}=0.30)$, but inversely with $b G S(P=0.05 ; b=-0.03)$. In the analysis excluding GA+, bGS directly associated with $\mathrm{P}+$ $(\mathrm{P}<0.0001 ; \mathrm{b}=2.007)$, PSA $(\mathrm{P}=0.04 ; \mathrm{b}=0.001)$, but inversely with $\mathrm{CII}+(\mathrm{P}=0.03 ; \mathrm{b}=-0.82)$. In the analysis excluding $\mathrm{CII}+$, bGS directly associated with $\mathrm{P}+(\mathrm{P}<$ $0.0001 ; b=2.06)$ and PSA $(P=0.05 ; b=0.001)$; however, there was no association with $G A+(P=0.31 ; b=-$ 0.36). In the subpopulation without PCA, CII+ directly associated with $\mathrm{GA}+(\mathrm{P}<0.0001 ; \mathrm{b}=0.39)$, but inversely with $\mathrm{GH}+(\mathrm{P}=0.02 ; \mathrm{b}=-0.18)$. In the analysis excluding $\mathrm{GA}+, \mathrm{CII}+$ inversely associated with $\mathrm{GH}+(\mathrm{P}=0.01$; $b=-0.23)$ and $P I N+(P=0.05 ; b=-0.56)$. In the analysis excluding $\mathrm{CII}+, \mathrm{GA}+$ inversely associated only with $\mathrm{PIN}+(\mathrm{P}=0.03 ; \mathrm{b}=-0.63)$.

Tables and Figure 1 are posted in Supplementary materials on www.aiua.it

\section{Discussion}

Our findings showed that, in a patient population undergoing prostate biopsy, chronic inflammation was independently and inversely associated with PCA. CII+ in men with positive biopsy cores was detected less frequently $(8.43 \%)$ than in those without $(25.78 \%)$; moreover, the OR of PCA in men with chronic inflammation was 0.26 . These findings suggest that, on biopsy cores, the presence of CII+ decrease the probability of detecting PCA by $76 \%$. We stress out that these findings indicate only an inverse independent association of CII+ with PCA; moreover, the relation does not necessary mean causation. The result of our investigation concord with previous studies discovering an evident inverse association between chronic inflammation and PCA $(4,7-9)$. As a result, CII+ might protect from the different steps involving genesis of cancer.

The relation between chronic inflammation and PCA was further investigated by our study which showed that, in the population, CII+ independently and inversely associated with HGPCA. As a result, CII+ in biopsy cores with HGPCA was found less frequently $(0.2 \%)$ than men without (4.8\%); moreover, the OR of HGPCA was 0.05. These results suggest that, on biopsy needle cores, the presence of CII+ decreases the probability of HGPCa by 95\%. Similar findings were detected in the PCA subpopulation. Indeed, CII+ in positive biopsy cores with HGPCA was found less frequently $(0.7 \%)$ than in those without chronic inflammation (14.1\%). The OR of HGPCA in CII+ was 0.13 which means that, in men with needle core biopsies positive for PCA, the probability of detecting HGPCA was decreased by $87 \%$; moreover, $\mathrm{CII}+$ was more common in needle biopsy cores with low intermediate grade cancers $(23.9 \%)$ than in those with HGPCA $(0.70 \%)$. These findings agree with the results of Zhang et al. who showed that chronic inflammation was more common in radical prostatectomy specimens with low grade tumours than in those with HGPCA (10). Once again; the protective association of CII+ should not be considered as causation; however, these findings sug- gest the chronic inflammation might protect from PCA progressing from low to high grade disease. Our data also showed that, in the PCA subpopulation, chronic inflammation was inversely and independently associated with a larger volume of percentage of positive biopsy cores.

Indeed, a CII+ was detected less frequently (6.3\%) in men with $\mathrm{P}_{+}>0.33$ than in patients without coexistent CII (41.6\%). The OR of PCA with $\mathrm{P}+>$ 0.33 in men with CII+ was 0.28 . This finding indicated that, on positive biopsy cores, the coexistence of chronic inflammation reduces the chance of having a proportion of $\mathrm{P}+>0.33$ by $72 \%$. Once again these findings, although not meaning causation, confirmed that there is inverse association between CII+ and PCA (4, 7-10).

The subject dealing with PCA associated with chronic inflammation has also been approached by other investigators, who, however, failed to detect any association (1113). Our investigation is a single centre study including a large number of patients collected consecutively in an appropriate time of interval (24 months); also, biopsy specimens have been evaluated by a dedicated pathologist, who routinely reports the presence or absence of CII+ in each biopsy core.

Moreover, our investigation, although consistent with other reports (4, 7-10), clearly shows that the coexistence of CII+ in needle biopsy specimens reduces the risk of aggressive prostate cancer.

This issue might have important drawbacks when approaching treatment options for PCA cancer such as active surveillance. It has been postulated that the exposure to non-steroidal anti-inflammatory drugs (NSAID) reduces the risk of cancer-genesis $(14,15)$. However, observational studies has shown that the PCA risk is increased after NSAID exposure $(16,17)$.

Moreover, cancer susceptibility and severity may be associated with functional polymorphisms of inflammatory cytokine genes, and deletion or inhibition of inflammatory cytokines inhibits the development of experimental cancer (18). Our study outlines the predictive role of chronic inflammation in PCA biology; it also supports the non appropriate role of NSAID exposure in prostate cancer genesis. Our results indicated that, in a subpopulation without PCA, chronic inflammation inversely associated with PSA $\leq$ Q1 (5.14 ug/l) which means that PSA serum levels $\leq$ Q1 were detected less frequently (10.3\%) in patients with coexistent CII+ in the biopsy specimen than in those without (25.3\%). The OR of 0.44 indicates that the presence of $\mathrm{CII}+$ in the specimens decreases the probability of detecting PSA serum levels $\leq$ Q1 by 56\%. These results agree with other investigations showing that chronic inflammation associates with elevated PSA serum levels (19-21).

Moreover, it has recently been reported that baseline prostate inflammation is associated with a reduced risk of PCA in men undergoing repeat prostate biopsy (22). As a theory, CII+ associates with increased PSA serum levels when there is contact and disruption of the glandular epithelium of the prostate.

There are limits in our study which was single centre and including only one dedicated pathologist. GA+ was not 
characterized according to the atrophy classification, proposed in 2006 by the working group for histology classification of prostate atrophy lesions which include simple atrophy, simple atrophy with cist formation, post atrophic hyperplasia and partial atrophy (23). The classification of low and high grade PIN was also not computed. Another limit of the present study may be related to the missed measurement of prostate volume with negative drawbacks on sampling procedures. Indeed biopsy procedures might not sample appropriately the large prostates with respect the smaller ones.

Finally, patients with inflammation may undergo to biopsy procedures more frequently than men without inflammation because of potential higher PSA levels.

\section{Conclusions}

There is an inverse negative association of chronic inflammation of the prostate type IV and risk of PCA. Chronic inflammation of the prostate type IV is less frequently detected in prostates with cancer. Moreover, HGPCA is less likely to be detected in cancers associated with coexistent CII. As a consequence, chronic inflammation of the prostate type IV might have important drawbacks for approaching and managing prostate diseases. Moreover, chronic inflammation in prostate microenvironment might be protective; however, the role of chronic inflammation in PCA carcinogenesis remains a controversial issue which needs further clinical and basic research.

\section{References}

1. Coussens LM, Werb Z. Inflammation and cancer. Nature. 2002; 420:860-67.

2. Mantovani A, Allavena P, Sica A, Balkwill F. Cancer related inflammation. Nature. 2008; 464:436-44.

3. Welson WG, De Marzo AM, Isaacs WB. Prostate Cancer. N Engl J Med. 2003; 349:366-81.

4. Blumenfeld W, Tucci S, Marayan P. Incidental lymphocytic prostatitis. Selective involvement with non malignant glands. AM J Surg Pathol. 1992; 16:975-81.

5. De Nunzio C, Kramer G, Marberger M, et al. The controversial relationship between benign prostatic hyperplasia and prostate cancer: the role of inflammation. Eur Urol. 2011; 60:106-17.

6. Krieger JN, Nyberg L Jr, Nickel JC. NIH consensus definition and classification of prostatitis. JAMA. 1999; 282:263-67.

7. Gerstenbluth RE, Seftel AD, Maclennan GT, et al. Distribution of chronic prostatitis in radical prostatectomy specimens with up-regulation of bcl-2 in areas of inflammation. J Urol. 2002; 167:2267-70.

8. Irani J, Goujon JM, Ragni et al. High-grade inflammation in prostate as a prognostic factor for biochemical recurrence after radical prostatectomy. Pathologist Multi Center Study Group. Urology. 1999; 54:467-72.

9. Karakiewicz PI, Benayoun S, Begin LR, et al. Chronic inflammation is negatively associated with prostate cancer and high-grade prostatic intraepithelial neoplasia on needle biopsy. Int J Clin Pract. 2007; 61:425-430

10. Zhang W, Sesterhenn IA, Connelly RR, et al. Inflammatory infiltrate (prostatitis) in whole mounted radical prostatectomy specimens from black and white patients is not an etiology for radical difference in prostate specific antigen. J Urol. 2000; 163:131-36.
11. Roberts RO, Bergstralh EJ, Bass SE, et al. Prostatitis as a risk factor for prostate cancer. Epidemiology. 2004; 15:93-99.

12. Davidsson S, Fiorentino M, Andren O, et al. Inflammation, focal atrophic lesions, and prostatic intraepithelial neoplasia with respect to risk of lethal prostate cancer. Cancer Epidemiol Biomarkers Prev. 2010; 20:2280-87.

13. Engelhardt PF, Brustmann H, Seklehner S, Riedl CR. Chronic asymptomatic inflammation of the prostate type IV and carcinoma of the prostate: is there a correlation? Scand J Urol. 2013; 47:230-5.

14. Thun MJ, Henley SJ, Patrono C. Nonsteroidal anti-inflammatory drugs as anticancers agents: mechanistic, pharmacologic, and clinical issues. J Natl Cancer Inst 2002; 94:252-66.

15. Gridley G, McLaughlin JK, Ekbom A et al. Incidence of cancer among patients with rheumatoid arthritis. J Nat. Cancer Inst. 1993; 85:307-11.

16. Langman MJ, Cheng KK, Gilman EA, Lancashire RJ. Effect antiinflammatory drugs on overall risk of common cancer: case-control study in general practice research data base. BMJ. 2000; 320:1462-66.

17. Neugut AI, Rosenberg DJ, Ahsan H, et al. Association between coronary heart disease and cancer of the breast, prostate and colon. Cancer Epidemiol Biomarkers Prev. 1998; 7:869-73.

18. Balkwill F, Mantovani A. Inflammation and cancer: back to Virchow? The Lancet. 2001; 357:539-45.

19. Hoekx L, Jeuris W, Van Marck E, Wyndaele JJ. Elevated serum prostate specific antigen (PSA) related to asymptomatic prostatic inflammation. Acta Urol Bel. 1998;66:1-2.

20. Sindhwani P, Wilson CM. Prostatis and serum prostate-specific antigen. Curr Urol Rep. 2005; 6:306-12.

(21) Hochreiter WW. The issue of elevated prostate cancer evaluation in men with elevated prostate-specific antigen and chronic prostatitis. Andrologia 2008; 40: 130-33.

12. Moreira DM, Nickel JC, Gerber L, et al. Baseline prostate inflammation is associated with a reduced risk of prostate cancer in men undergoing repeat prostate biopsy. Cancer. 00, 2013.

23. De Marzo AM, Platz EA, Epstein JI, et al. A working group classification of focal prostate atrophy lesions. Am J Surg Pathol. 2006; 30:1281-91.

\section{Correspondence}

Antonio Benito Porcaro, MD - drporcaro@yahoo.com

Filippo Migliorini, MD

Stefano Zecchini Antoniolli, MD

Vincenzo Lacola, MD

Carmelo Monaco, MD

Pierpaolo Curti, MD

Stefano Cavalleri, MD

Romeo Pianon, $M D$

Walter Artibani, MD, Professor

Azienda Ospedaliera Universitaria Integrata Verona,

Dipartimento ad Attività Integrata di Chirurgia ed Oncologia, Pancreas Center, Divisione Clinicizzata di Urologia, Policlinico GB Rossi

P.le LA Scuro, 10 - 37134 Verona, Italy

Emanuele Rubilotta, $M D$

Aldo Petrozziello, MD

Dpt. Geriatric Medicine/Endocrinology, Azienda Ospedaliera

Universitaria Integrata, Ospedale Policlinico and Ospedale Civile Maggiore, Verona, Italy

Claudio Ghimenton, MD

Dpt. Pathology, Azienda Ospedaliera Universitaria Integrata Ospedale Policlinico and Ospedale Civile Maggiore, Verona, Italy 